\title{
HOW POROUS IS THE GRAPH OF BROWNIAN MOTION?
}

\author{
J. T. COX AND PHILIP S. GRIFFIN
}

\begin{abstract}
We prove that the graph of Brownian motion is almost surely porous, and determine the Hausdorff dimension of sets with a given porosity index. In particular we show that the porosity index of the graph is $\gamma_{0} \doteq 0.6948$.
\end{abstract}

\section{INTRODUCTION AND STATEMENT OF RESULTS}

Real function theory has seen an increase in interest recently in the study of porous and $\sigma$-porous sets. These are sets which are "small" in a certain sense. They were first introduced by Denjoy [4] and later rediscovered by Dolzhenko [5]. Much of the recent interest centers around the work of Zajicek [12]. For further references see [6 and 11].

Since we are primarily interested in the graph of Brownian motion, we will only define porosity for the graph of a real valued function. Given $f:[0, \infty) \rightarrow$ $\mathbb{R}$ let $G$ be its graph, $G=\{(t, f(t)): t \geq 0\}$. Using $\Lambda$ to denote a square in $\mathbb{R}^{2}$ with sides parallel to the coordinate axes, we define

$$
\Lambda_{t}(h)=\{(x, s): t-h \leq s \leq t+h, f(t)-h \leq x \leq f(t)+h\},
$$

and

$$
\delta_{t}(h)=\sup \left\{|\Lambda|^{1 / 2}: \Lambda \subseteq \Lambda_{t}(h), \Lambda \cap G=\varnothing\right\},
$$

where $|\cdot|$ denotes Lebesgue measure. Thus $\delta_{t}(h)$ is the supremum of the side lengths of all squares contained in $\Lambda_{t}(h)$, with sides parallel to the coordinate axes, which do not intersect $G$. The function $f$, or more precisely its graph $G$, is said to be porous at $t$ if

$$
\limsup _{h \downarrow 0} \frac{\delta_{t}(h)}{h}>0 .
$$

It is porous if (1.1) holds for all $t \geq 0$. The definition of porosity from the right is obtained by replacing $\Lambda_{t}(h)$ and $\delta_{t}(h)$ with

$$
\Lambda_{t}^{+}(h)=\{(s, x): t \leq s \leq t+h, f(t)-h \leq x \leq f(t)+h\}
$$

Received by the editors April 10, 1989.

1980 Mathematics Subject Classification (1985 Revision). Primary 60J65, 60G17.

Key words and phrases. Brownian motion, arcsine law, porous sets, Hausdorff dimension.

Research of the first author supported in part by NSF Grant DMS-8802055 and research of the second author supported in part by NSF Grant DMS-8700928. 
and

$$
\delta_{t}^{+}(h)=\sup \left\{|\Lambda|^{1 / 2}: \Lambda \subseteq \Lambda_{t}^{+}(h), \Lambda \cap G=\varnothing\right\}
$$

respectively. The definition of porosity from the left should be clear.

Our original interest in porosity is due to the following question raised by Goffman [7]:

Is the graph of Brownian motion porous with probability one?

More precisely, let $W(t), t \geq 0$, be a standard one-dimensional Brownian motion, and let $\delta_{t}$ be defined for the function $f(t)=W(t)$. The question is then whether or not

$$
P\left(\limsup _{h \downarrow 0} \frac{\delta_{t}(h)}{h}>0 \text { for all } t \geq 0\right)=1 .
$$

The analogous question for porosity from the right is whether or not

$$
P\left(\limsup _{h \downarrow 0} \frac{\delta_{t}^{+}(h)}{h}>0 \text { for all } t \geq 0\right)=1 \text {. }
$$

As we will see the analysis of porosity for Brownian motion essentially reduces to the analysis of right porosity, and thus we turn out attention to this first.

We will show that the answer to Goffman's question is yes by showing that (1.3) holds. Our original proof in fact gave the stronger result that there exists a (nonrandom) $\varepsilon>0$ for which

$$
P\left(\limsup _{h \downarrow 0} \frac{\delta_{t}^{+}(h)}{h} \geq \varepsilon \text { for all } t \geq 0\right)=1 .
$$

This then raises the question as to the largest value of $\varepsilon$ for which (1.4) holds. This value is called the right porosity index of the graph. Analogously the value of the limsup in (1.1), with $\delta_{t}$ replaced by $\delta_{t}^{+}$, is called the right porosity index at $t$.

Before addressing this problem we will make a few simple observations. First, it follows from the definitions that one always has for every $h>0$

$$
\delta_{t}^{+}(h) / h \leq 1 \text {. }
$$

It is not hard to show (see the end of $\S 3$ ) that for every $t \geq 0$

$$
P\left(\limsup _{h \downarrow 0} \frac{\delta_{t}^{+}(h)}{h}=1\right)=1 \text {. }
$$

Hence by Fubini

$$
P\left(\limsup \frac{\delta_{t}^{+}(h)}{h}=1 \text { for a.e. } t\right)=1 .
$$

Thus the right porosity index at almost all $t$ is 1 with probability one. However, the value of the right porosity index of the graph is not 1 , as can be seen from our first result. 
Theorem 1.1. $P\left(\inf _{t \geq 0} \lim \sup _{h \downarrow 0} \delta_{t}^{+}(h) / h=\frac{1}{2}\right)=1$

From Theorem 1.1 and (1.7) we see that

$$
A^{+}=\left\{t: \limsup _{h \downarrow 0} \frac{\delta_{t}^{+}(h)}{h}<1\right\}
$$

satisfies

$$
P\left(A^{+} \neq \varnothing\right)=1 \text { and } P\left(|A|^{+}=0\right)=1 \text {. }
$$

Thus $A^{+}$is a set of one-dimensional Lebesgue measure zero but it is still quite "large". More precisely, if for any set $B$ we let

$$
d(B)=\text { Hausdorff dimension of } B,
$$

then we are able to show

Theorem 1.2. $P\left(d\left(A^{+}\right)=1\right)=1$.

Our final result on right porosity concerns the size of the set

$$
A_{\gamma}^{+}=\left\{t: \limsup _{h \downarrow 0} \frac{\delta_{t}^{+}(h)}{h} \leq \gamma\right\}
$$

where $0 \leq \gamma<1$. If $\gamma<\frac{1}{2}$ then $A_{\gamma}^{+}=\varnothing$ by Theorem 1.1. If $\gamma \geq \frac{1}{2}$ we again ask for its Hausdorff dimension. To answer this question, we define $\alpha(\gamma)$ for $\gamma \in(0,1)$ to be the unique solution $\alpha$ of

$$
\int_{1}^{(1-\gamma)^{-1}} \frac{t^{\alpha}-1}{(t-1)^{\frac{3}{2}}} d t=2\left(\frac{1-\gamma}{\gamma}\right)^{\frac{1}{2}} .
$$

This expression has its origin in the determination of the moments of a certain stopping time (see Lemma 2.1 below). We then have

Theorem 1.3. For $\frac{1}{2} \leq \gamma<1$

$$
P\left(d\left(A_{\gamma}^{+}\right)=1-\alpha(\gamma)\right)=1 \text {. }
$$

To state the analogous results to Theorems $1.1-1.3$ for porosity, define

$$
A=\left\{t: \limsup _{h \downarrow 0} \frac{\delta_{t}(h)}{h}<1\right\}
$$

and

$$
A_{\gamma}=\left\{t: \limsup _{h \downarrow 0} \frac{\delta_{t}(h)}{h} \leq \gamma\right\}
$$


We then have

Theorem 1.4. Let $\gamma_{0} \doteq 0.6948$ be the unique value of $\gamma$ for which (1.10) holds with $\alpha=\frac{1}{2}$. Then

$$
P\left(\inf _{t \geq 0} \limsup _{h \downarrow 0} \frac{\delta_{t}(h)}{h}=\gamma_{0}\right)=1
$$

Theorem 1.5. $P(d(A)=1)=1$.

Theorem 1.6. For $\gamma_{0} \leq \gamma<1$

$$
P\left(d\left(A_{\gamma}\right)=1-2 \alpha(\gamma)\right)=1 .
$$

We are also able to obtain the Hausdorff dimension of the intersection of $A_{\gamma}$ and $A_{\gamma}^{+}$with an arbitrary analytic set. These results are stated in $\S 2$, where we will reformulate our results in a more convenient form (see Theorems 2.1 and 2.2). The proofs are given in $\S \S 3$ and 4 .

In view of (1.6), Theorems 1.1-1.6 can be considered as results concerning points on the graph of Brownian motion which are "less porous" than they should be. The study of such points turns out to be closely related to the study of Brownian slow points (see [1-3, 8-10]). In particular, the methods developed by Davis in [2] for finding slow points, and Perkins in [10] for computing Hausdorff dimensions, play a very important role in our analysis. The reader familiar with their work will note the close correspondence between the statements of our results and those in [10] on which they are modelled.

\section{Preliminaries}

Recall that $W(s)$ is a standard one-dimensional Brownian motion. For $\beta>$ 1 and $\varepsilon \in(0, \beta-1)$ let $Z(\varepsilon, \beta)$ be a random variable with distribution given by

$$
\begin{gathered}
P(Z(\varepsilon, \beta)=0)=\frac{2}{\pi} \operatorname{arcsine}\left(\frac{\varepsilon}{\beta-1}\right)^{1 / 2}, \\
P(1+\varepsilon \leq Z(\varepsilon, \beta) \leq t)=1-\frac{2}{\pi} \operatorname{arcsine}\left(\frac{\varepsilon}{t-1}\right)^{1 / 2}, \quad t \in[1+\varepsilon, \beta] .
\end{gathered}
$$

The way in which this distribution arises is as follows; fix $h>0$ and let

$$
\tau=\inf \{s \geq(1+\varepsilon) h: W(s)=W(h)\} .
$$

Then the arcsine law and scaling imply that

$$
Z(\varepsilon, \beta) \stackrel{d}{=} \frac{\tau}{h} 1\left(\frac{\tau}{h} \leq \beta\right),
$$

where we have used $\stackrel{d}{=}$ to denote equality in distribution, and $1(\cdot)$ for indicator function.

Differentiating in $(2.1)$, one sees that the density of $Z(\varepsilon, \beta)$ on $[1+\varepsilon, \beta]$ is given by

$$
f(t)=\frac{1}{\pi}\left(\frac{\varepsilon}{t-1-\varepsilon}\right)^{1 / 2} \frac{1}{t-1} .
$$


Thus for $p \geq 0$

$$
E\left[Z^{p}(\varepsilon, \beta)\right]=\frac{\varepsilon^{1 / 2}}{\pi} \int_{1+\varepsilon}^{\beta} \frac{t^{p}}{t-1} \frac{d t}{(t-1-\varepsilon)^{1 / 2}} .
$$

Lemma 2.1. Fix $p \geq 0$ and $\beta>1$. Then

$$
E\left[Z^{p}(\varepsilon, \beta)\right]=1+\varepsilon^{1 / 2} \frac{1}{\pi}\left[\int_{1}^{\beta} \frac{t^{p}-1}{(t-1)^{3 / 2}} d t-\frac{2}{(\beta-1)^{1 / 2}}\right]+o\left(\varepsilon^{1 / 2}\right)
$$

as $\varepsilon \rightarrow 0$.

Proof. For any $p \geq 0$ and $\beta>1$,

$$
\begin{aligned}
E\left[Z^{p}(\varepsilon, \beta)\right] & =\frac{\varepsilon^{1 / 2}}{\pi} \int_{1+\varepsilon}^{\beta} \frac{t^{p}-1}{(t-1)} \frac{d t}{(t-1-\varepsilon)^{1 / 2}}+\frac{\varepsilon^{1 / 2}}{\pi} \int_{1+\varepsilon}^{\beta} \frac{1}{t-1} \frac{d t}{(t-1-\varepsilon)^{1 / 2}} \\
& =\mathrm{I}+\mathrm{II} .
\end{aligned}
$$

An application of dominated convergence shows that

$$
\int_{1+\varepsilon}^{\beta} \frac{t^{p}-1}{t-1} \frac{d t}{(t-1-\varepsilon)^{1 / 2}} \rightarrow \int_{1}^{\beta} \frac{t^{p}-1}{(t-1)^{3 / 2}} d t<\infty
$$

as $\varepsilon \rightarrow 0$. Thus

$$
\mathrm{I}=\frac{\varepsilon^{1 / 2}}{\pi} \int_{1}^{\beta} \frac{t^{p}-1}{(t-1)^{3 / 2}} d t+o\left(\varepsilon^{1 / 2}\right) .
$$

The integral in II can be explicitly evaluated, giving

$$
\begin{aligned}
\text { II } & =1-\frac{2}{\pi} \operatorname{arcsine}\left(\frac{\varepsilon}{\beta-1}\right)^{1 / 2} \\
& =1-\frac{2}{\pi}\left(\frac{\varepsilon}{\beta-1}\right)^{1 / 2}+o\left(\varepsilon^{1 / 2}\right) .
\end{aligned}
$$

Combining (2.2) and (2.3) finishes the proof.

Now fix $\beta>1$ and define

$$
g(p)=\int_{1}^{\beta} \frac{t^{p}-1}{(t-1)^{3 / 2}} d t, \quad p \geq 0
$$

Clearly $g$ is continuous, strictly increasing, $g(0)=0$, and $g(p) \rightarrow \infty$ as $p \rightarrow \infty$. In particular, for each $\beta \in(1, \infty)$, there exists a unique $p(\beta)$ such that

$$
g(p(\beta))=2(\beta-1)^{-1 / 2} .
$$

The following observations will be used frequently throughout the paper:

(i) $p(\cdot)$ is continuous,

(ii) $p(\cdot)$ is strictly decreasing,

(iii) $p(\beta) \uparrow \infty$ as $\beta \downarrow 1, p(\beta) \downarrow 0$ as $\beta \uparrow \infty$,

(iv) $p(2)=1$,

(v) if $p_{1}<p(\beta)<p_{2}$, then for all $\varepsilon>0$ sufficiently small $E\left[Z^{p_{1}}(\varepsilon, \beta)\right]<1$ and $E\left[Z^{p_{2}}(\varepsilon, \beta)\right]>1$. 
Properties (i)-(iv) are all easy consequences of the definition of $p(\beta)$, and (v) follows from Lemma 2.1.

Observe that (1.10) is just a reparameterization of (2.5) obtained by setting $\gamma=1-\beta^{-1}$. It is more convenient however to work directly with $p(\beta)$ and so we now reformulate our results in these terms. For $\beta \in(1, \infty)$ define

$$
\begin{aligned}
& B_{\beta}^{+}=\left\{t \in[0,1]: \limsup _{h \downarrow 0} \frac{\delta_{t}^{+}(h)}{h} \leq 1-\beta^{-1}\right\}, \\
& B_{\beta}=\left\{t \in[0,1]: \limsup _{h \downarrow 0} \frac{\delta_{t}(h)}{h} \leq 1-\beta^{-1}\right\} .
\end{aligned}
$$

We will prove

Theorem 2.1. Let $B \subseteq[0,1]$ be an analytic set, then

$$
\begin{aligned}
& P\left(d\left(B \cap B_{\beta}^{+}\right)=d(B)-p(\beta)\right)=1 \quad \text { if } p(\beta) \leq d(B), \\
& P\left(B \cap B_{\beta}^{+}=\varnothing\right)=1 \quad \text { if } p(\beta)>d(B) .
\end{aligned}
$$

Theorem 2.2. Let $B \subseteq[0,1]$ be an analytic set, then

$$
\begin{aligned}
& P\left(d\left(B \cap B_{\beta}\right)=d(B)-2 p(\beta)\right)=1 \quad \text { if } 2 p(\beta) \leq d(B), \\
& P\left(B \cap B_{\beta}=\varnothing\right)=1 \quad \text { if } 2 p(\beta)>d(B) .
\end{aligned}
$$

In Theorems 2.1 and 2.2 we consider $t \in[0,1]$; it follows from the Markov property that the same conclusions hold for $t \in[n, n+1]$ for any positive integer $n$, and hence for $t \in[0, \infty)$. Thus, Theorem 1.1 follows from Theorem 2.1 by using (2.6)(ii) and (iv); Theorem 1.2 follows from Theorem 2.1 by letting $\beta \rightarrow \infty$ and using (2.6)(iii); and Theorem 1.3 is just a special case of Theorem 2.1. Similarly, Theorems 1.4-1.6 follow from Theorem 2.2.

\section{UPPER BOUNDS ON $d\left(B \cap B_{\beta}^{+}\right)$AND $d\left(B \cap B_{\beta}\right)$}

For $\eta>0$ define

$$
\begin{aligned}
& B_{\beta, \eta}^{+}=\left\{t \in[0,1]: \frac{\delta_{t}^{+}(h)}{h} \leq\left(1-\beta^{-1}\right) \text { for all } h \in(0, \eta]\right\}, \\
& B_{\beta, \eta}^{-}=\left\{t \in[0,1]: \frac{\delta_{t}^{-}(h)}{h} \leq\left(1-\beta^{-1}\right) \text { for all } h \in(0, \eta]\right\},
\end{aligned}
$$

and

$$
\begin{array}{r}
B_{\varepsilon, \beta, \eta}^{+}=\left\{t \in[0,1]: \forall h \in\left(0, \eta \beta^{-1}\right] \exists s \in[(1+\varepsilon) h, \beta h]\right. \\
\text { with } W(t+s)=W(t)\}, \\
B_{\varepsilon, \beta, \eta}^{-}=\left\{t \in[0,1]: \forall h\left(0, \eta \beta^{-1}\right] \exists s \in[(1+\varepsilon) h, \beta h]\right. \\
\text { with } W(t-s)=W(t)\} .
\end{array}
$$


In order that the definition of $B_{\varepsilon, \beta, \eta}^{-}$make sense we adopt the convention that $W(u)=-\infty$ for $u<0$. For $\eta_{1}<\eta_{2}$

$$
B_{\beta, \eta_{1}}^{+} \supseteq B_{\beta, \eta_{2}}^{+}
$$

while for $\beta_{1}<\beta_{2}$

$$
\lim _{\eta \downarrow 0} B_{\beta_{1}, \eta}^{+} \subseteq B_{\beta_{1}}^{+} \subseteq \lim _{\eta \downarrow 0} B_{\beta_{2}, \eta}^{+} .
$$

The following observation is central to our methods for obtaining bounds on the size of $B_{\beta, \eta}^{+}$and hence on $B_{\beta}^{+}$: if $\beta_{1}<\beta_{2}$, and $\varepsilon>0$ is small enough that $1-(1+\varepsilon) \beta_{2}^{-1}>1-\beta_{1}^{-1}$, then

$$
B_{\beta_{1}, \eta}^{+} \subseteq B_{\varepsilon, \beta_{2}, \eta}^{+} \text {. }
$$

To prove this, suppose $t \notin B_{\varepsilon, \beta_{2}, \eta}^{+}$, and hence that for some $h \in\left(0, \eta \beta_{2}^{-1}\right]$ we have $W(t+s) \neq W(t)$ for all $s \in\left[(1+\varepsilon) h, \beta_{2} h\right]$. Then either

$$
\Lambda=\left\{(t+s, x):(1+\varepsilon) h \leq s \leq \beta_{2} h \text { and } W(t)-\left(\beta_{2}-(1+\varepsilon)\right) h \leq x \leq W(t)\right\}
$$

or

$$
\Lambda=\left\{(t+s, x):(1+\varepsilon) h \leq s \leq \beta_{2} h \text { and } W(t) \leq x \leq W(t)+\left(\beta_{2}-(1+\varepsilon)\right) h\right\}
$$

satisfies

$$
\Lambda \subseteq \Lambda_{t}^{+}\left(\beta_{2} h\right) \backslash G
$$

by continuity of the sample paths $(G$ is the graph of $W(s)$ ). Consequently, $h^{\prime}=\beta_{2} h \in(0, \eta]$ satisfies

$$
\frac{\delta_{t}^{+}\left(h^{\prime}\right)}{h^{\prime}}=\frac{\delta_{t}^{+}\left(\beta_{2} h\right)}{\beta_{2} h} \geq \frac{\beta_{2}-(1+\varepsilon)}{\beta_{2}}>1-\beta_{1}^{-1} .
$$

So $t \notin B_{\beta_{1}, \eta}^{+}$, and (3.4) follows.

Similarly, the analogous versions of (3.2)-(3.4) for $B_{\beta}^{-}, B_{\beta, \eta}^{-}$, and $B_{\varepsilon, \beta, \eta}^{-}$ are valid. We now give an estimate on the size of $B_{\varepsilon, \beta, \eta}^{+}$. Let

$$
\mathscr{F}(t)=\sigma\{W(s): 0 \leq s \leq t\} .
$$

Proposition 3.1. Fix $\beta>1, \varepsilon \in(0, \beta-1)$ and $p \geq 0$, and assume that $E\left[Z^{p}(\varepsilon, \beta)\right]<1$. Then for any $\eta>0$ there exists $C<\infty$ such that for all $v \geq 0$ and all $\zeta$ sufficiently small,

$$
P\left([v, v+\zeta] \cap B_{\varepsilon, \beta, \eta}^{+} \neq 0 \mid \mathscr{F}(v+\zeta)\right) \leq C \zeta^{p} \quad \text { a.s. }
$$

Proof. By the Markov property, it suffices to consider the case $v=0$. Choose $q>p$ and $\beta_{1}>\beta$ such that $E\left[Z^{q}\left(\varepsilon, \beta_{1}\right)\right]<1$. Fix $\lambda \in(0,1)$ such that $\lambda q \geq p$, and assume that

$$
\zeta \leq\left(\frac{\beta_{1}-\beta}{\beta-1}\right)^{1 /(1-\lambda)} \wedge \frac{1}{2} .
$$


Finally let $r_{\zeta}=(-2 \zeta p \log \zeta)^{1 / 2}$. Then with $M(t)=\sup \{|W(s)|: 0 \leq s \leq t\}$ we have

$$
\begin{aligned}
& P\left([0, \zeta] \cap B_{\varepsilon, \beta, \eta}^{+} \neq \varnothing \mid \mathscr{F}(\zeta)\right) \\
& \leq P\left(\exists b \in(-\infty, \infty) \forall h \in\left(\zeta, \eta \beta^{-1}\right] \exists s\right. \\
& \in[(1+\varepsilon) h, \beta h] \text { with } W(s)=b \mid \mathscr{F}(\zeta)) \\
& \leq P\left(\exists b \in(-\infty, \infty) \forall h \in\left(2 \zeta, \eta \beta^{-1}\right] \exists s\right. \\
& \in[(1+\varepsilon) h, \beta h] \text { with } W(s)=b \mid \mathscr{F}(\zeta)) \\
& =P\left(\exists b \in(-\infty, \infty) \forall h \in\left(\zeta, \eta \beta^{-1}-\zeta\right] \exists s\right. \\
& \in[(1+\varepsilon)(h+\zeta)-\zeta, \beta(h+\zeta)-\zeta] \text { with } W(s)=b) \text { a.s. } \\
& \leq P\left(M(\zeta) \geq r_{\zeta}\right) \\
& +P\left(\exists b,|b| \leq r_{\zeta} \text { such that } \forall h \in\left(\zeta, \eta \beta^{-1}-\zeta\right]\right. \text {, } \\
& \exists s \in[(1+\varepsilon)(h+\zeta)-\zeta, \beta(h+\zeta)-\zeta] \text { with } W(s)=b) \\
& =\mathrm{I}+\mathrm{II} \text {. }
\end{aligned}
$$

We will estimate I and II separately.

The first term is simple:

$$
\begin{aligned}
\mathrm{I} & \leq 4 P\left(W(\zeta) \geq r_{\zeta}\right)=4 P\left(W(1) \geq \zeta^{-1 / 2} r_{\zeta}\right) \\
& \leq 2(-\pi p \log \zeta)^{-1 / 2} \exp (p \log \zeta) \leq C \zeta^{p}
\end{aligned}
$$

for some $C$.

To bound the second term we use scaling to obtain,

$$
\begin{aligned}
\mathrm{II} & \leq P\left(\forall h \in\left[\zeta^{\lambda}, \eta \beta^{-1}-\zeta\right] \exists s \in[(1+\varepsilon)(h+\zeta)-\zeta, \beta(h+\zeta)-\zeta]\right. \\
& =P\left(\forall u \in\left[1, \zeta^{-\lambda}\left(\eta \beta^{-1}-\zeta\right)\right] \exists s \in\left[(1+\varepsilon) u+\varepsilon \zeta^{1-\lambda}, \beta u+(\beta-1) \zeta^{1-\lambda}\right]\right. \\
& \text { with } \left.|W(s)| \leq r_{\zeta}\right) \\
& \leq P\left(\forall u \in\left[1, \zeta^{-\lambda}\left(\eta \beta^{-1}-\zeta\right)\right] \exists s \in\left[(1+\varepsilon) u, \beta_{1} u\right] \text { with }|W(s)| \leq \varepsilon_{\zeta}\right) \\
& =P\left(\Gamma_{\zeta}\right)
\end{aligned}
$$

say, where $\varepsilon_{\zeta} \stackrel{\text { def }}{=} \zeta^{-\lambda / 2} r_{\zeta} \rightarrow 0$ as $\zeta \downarrow 0$. To estimate this last quantity we introduce stopping times $\tau_{k}$, which are finite a.s., defined by

$$
\tau_{-1}=1, \quad \tau_{k}=\inf \left\{s \geq(1+\varepsilon) \tau_{k-1}:|W(s)| \leq \varepsilon_{\zeta}\right\}, \quad k \geq 0 .
$$

Also define

$$
\begin{aligned}
V_{k}\left(\varepsilon, \beta_{1}\right) & =\frac{\tau_{k}}{\tau_{k-1}} 1\left(\frac{\tau_{k}}{\tau_{k-1}} \leq \beta_{1}\right), \quad k \geq 0, \\
V^{*}\left(\varepsilon, \beta_{1}\right) & =\sup _{k \geq 0} \prod_{m=0}^{k} V_{m}\left(\varepsilon, \beta_{1}\right) .
\end{aligned}
$$


Observe that

$$
\Gamma_{\zeta} \subset\left\{V^{*}\left(\varepsilon, \beta_{1}\right) \geq \zeta^{-\lambda}\left(\eta \beta^{-1}-\zeta\right)\right\}
$$

Now if $u \geq 1$ and $|x| \leq \varepsilon_{\zeta}$, then for any $t \in\left[1+\varepsilon, \beta_{1}\right]$ and any $k \geq 0$

$$
\begin{aligned}
& P\left(V_{k+1}\left(\varepsilon, \beta_{1}\right) \in[1+\varepsilon, t] \mid \tau_{k}=u, W\left(\tau_{k}\right)=x\right) \\
& \quad=P\left(\exists s \in[(1+\varepsilon) u, t u] \text { with }|W(s)| \leq \varepsilon_{\zeta} \mid W(u)=x\right) \\
& \quad=P\left(\exists s \in[(1+\varepsilon), t] \text { with }|W(s)| \leq \varepsilon_{\zeta} u^{-1 / 2} \mid W(1)=x u^{-1 / 2}\right) \\
& \quad \rightarrow P\left(Z\left(\varepsilon, \beta_{1}\right) \in[1+\varepsilon, t]\right)
\end{aligned}
$$

uniformly in $u, x$, and $k$ as $\zeta \downarrow 0$. Since the random variables are bounded, this means that for all $\alpha \geq 0$, all $k \geq 0$ and all $\delta>0$

$$
E\left[V_{k+1}^{\alpha}\left(\varepsilon, \beta_{1}\right) \mid \mathscr{F}\left(\tau_{k}\right)\right] \leq E\left[Z^{\alpha}\left(\varepsilon, \beta_{1}\right)\right]+\delta \text { a.s. }
$$

provided $\zeta$ is sufficiently small. In particular since $E\left[Z^{q}\left(\varepsilon, \beta_{1}\right)\right]<1$, there exists an $r<1$ such that for all $\zeta$ sufficiently small and all $k \geq 0$

$$
E\left[V_{k+1}^{q}\left(\varepsilon, \beta_{1}\right) \mid \mathscr{F}\left(\tau_{k}\right)\right] \leq r \quad \text { a.s. }
$$

Thus by the strong Markov property, for any $j \geq 1$ and $\zeta$ small

$$
E\left[\prod_{m=0}^{j} V_{m}^{q}\left(\varepsilon, \beta_{1}\right)\right]=E E\left[\prod_{m=0}^{j} V_{m}^{q}\left(\varepsilon, \beta_{1}\right) \mid \mathscr{F}\left(\tau_{j-1}\right)\right] \leq r E\left[\prod_{m=0}^{j-1} V_{m}^{q}\left(\varepsilon, \beta_{1}\right)\right] \text {. }
$$

Hence, since $V_{0}\left(\varepsilon, \beta_{1}\right) \leq \beta_{1}$, we have for all small $\zeta$

$$
E\left[V^{*}\left(\varepsilon, \beta_{1}\right)^{q}\right] \leq \sum_{k=0}^{\infty} E\left[\prod_{m=0}^{k} V_{m}^{q}\left(\varepsilon, \beta_{1}\right)\right] \leq \beta_{1}^{q} \sum_{k=0}^{\infty} r^{k}<\infty
$$

Thus by (3.6)

for some constant $C$.

$$
P\left(\Gamma_{\zeta}\right) \leq \frac{E\left[V^{*}\left(\varepsilon, \beta_{1}\right)^{q}\right]}{\zeta^{-\lambda q}\left(\eta \beta^{-1}-\zeta\right)^{q}} \leq C \zeta^{p}
$$

We note that by integration in (3.5), for all $v \geq 0$ and all $\zeta$ sufficiently small

$$
P\left([v, v+\zeta] \cap B_{\varepsilon, \beta, \eta}^{+} \neq \varnothing\right) \leq C \zeta^{p},
$$

and by considering the Brownian motion $\widetilde{W}(s)=W(1)-W(1-s)$, that

$$
P\left([v, v+\zeta] \cap B_{\varepsilon, \beta, \eta}^{-} \neq \varnothing\right) \leq C \zeta^{p} .
$$

With Proposition 3.1 at hand, the proof of Theorem 3.1 is now similar to that of the upper bound in Theorem 4 of Perkins [10].

Theorem 3.1. Assume $B \subseteq[0,1]$. Then

$$
\begin{gathered}
P\left(d\left(B \cap B_{\beta_{1}}^{+}\right) \leq d(B)-p\left(\beta_{1}\right)\right)=1, \quad d(B) \geq p\left(\beta_{1}\right) \\
P\left(B \cap B_{\beta_{1}}^{+}=\varnothing\right)=1, \quad d(B)<p\left(\beta_{1}\right)
\end{gathered}
$$


and

(3.8)

$$
\begin{gathered}
P\left(d\left(B \cap B_{\beta_{1}}^{+} \cap B_{\beta_{2}}^{-}\right) \leq d(B)-p\left(\beta_{1}\right)-p\left(\beta_{2}\right)\right)=1, \quad d(B) \geq p\left(\beta_{1}\right)+p\left(\beta_{2}\right) ; \\
P\left(B \cap B_{\beta_{1}}^{+} \cap B_{\beta_{2}}^{-}=\varnothing\right)=1, \quad d(B)<p\left(\beta_{1}\right)+p\left(\beta_{2}\right) .
\end{gathered}
$$

Proof. We start with the proof of (3.8). For $i=1,2$ fix $p_{i}<p\left(\beta_{i}\right)$, and if $d(B)<p\left(\beta_{1}\right)+p\left(\beta_{2}\right)$ let the $p_{i}$ also satisfy $p_{1}+p_{2}>d(B)$. By (2.6) we can find $\xi_{i}>\beta_{i}$ so that $p_{i}<p\left(\xi_{i}\right)<p\left(\beta_{i}\right)$ for $i=1,2$. We can also choose $\varepsilon>0$ sufficiently small such that

$$
1-(1+\varepsilon) \xi_{i}^{-1}>1-\beta_{i}^{-1}
$$

and

$$
E\left[Z^{p_{i}}\left(\varepsilon, \xi_{i}\right)\right]<1 .
$$

It follows from (3.3) and (3.4) that

$$
B_{\beta_{i}}^{+} \subseteq \lim _{\eta \downarrow 0} B_{\varepsilon, \xi_{i}, \eta}^{+}
$$

with the analogous result holding if + is replaced by - .

Next, fix $\lambda>d(B)$ (if $d(B)<p\left(\beta_{1}\right)+p\left(\beta_{2}\right)$ let $\lambda=p_{1}+p_{2}$ ). For $n$ sufficiently large we can choose a cover $\left\{S_{i}\right\}$ of $B$ such that

$$
\rho\left(S_{i}\right) \leq n^{-1} \forall i, \quad \text { and } \quad \sum_{1}^{\infty} \rho\left(S_{i}\right)^{\lambda} \leq n^{-1},
$$

where $\rho(S)=\sup \{|x-y|: x, y \in S\}$ is the diameter of $S$.

Now consider the cover of $B \cap B_{\varepsilon, \xi_{1}, \eta}^{+} \cap B_{\varepsilon, \xi_{2}, \eta}^{-}$given by $\left\{\widetilde{S}_{i}\right\}$, where

$$
\widetilde{S}_{i}= \begin{cases}S_{i}, & \text { if } S_{i} \cap B_{\varepsilon, \xi_{1}, \eta}^{+} \cap B_{\varepsilon, \xi_{2}, \eta}^{-} \neq \varnothing, \\ \varnothing, & \text { otherwise. }\end{cases}
$$

Note that

$$
E\left[\sum_{i=1}^{\infty} \rho\left(\widetilde{S}_{i}\right)^{\lambda-p_{1}-p_{2}}\right]=\sum_{i=1}^{\infty} \rho\left(S_{i}\right)^{\lambda-p_{1}-p_{2}} P\left(S_{i} \cap B_{\varepsilon, \xi_{1}, \eta}^{+} \cap B_{\varepsilon, \xi_{2}, \eta}^{-} \neq \varnothing\right) .
$$

Since $S_{i} \subseteq\left[v_{i}, v_{i}+s_{i}\right]$ for some $v_{i}$, where $s_{i}=\rho\left(S_{i}\right)$, we have for sufficiently large $n$

$$
\begin{aligned}
P\left(S_{i}\right. & \left.\cap B_{\varepsilon, \xi_{1}, \eta}^{+} \cap B_{\varepsilon, \xi_{2}, \eta}^{-} \neq \varnothing\right) \\
& \leq E\left[E\left[1\left(S_{i} \cap B_{\varepsilon, \xi_{2}, \eta}^{-} \neq \varnothing\right) 1\left(S_{i} \cap B_{\varepsilon, \xi_{1}, \eta}^{+} \neq \varnothing\right) \mid \mathscr{F}\left(v_{i}+s_{i}\right)\right]\right] \\
& =E\left[1\left(S_{i} \cap B_{\varepsilon, \xi_{2}, \eta}^{-} \neq \varnothing\right) P\left(S_{i} \cap B_{\varepsilon, \xi_{1}, \eta}^{+} \neq \varnothing \mid \mathscr{F}\left(v_{i}+s_{i}\right)\right)\right] \\
& \leq C \rho\left(S_{i}\right)^{p_{1}} P\left(S_{i} \cap B_{\varepsilon, \xi_{2}, \eta}^{-} \neq \varnothing\right) \\
& \leq C^{2} \rho\left(S_{i}\right)^{p_{1}+p_{2}}
\end{aligned}
$$


by Proposition 3.1 and the remarks following it. Hence by (3.12) and Fatou

$$
\liminf _{n \rightarrow \infty} \sum_{i=1}^{\infty} \rho\left(\widetilde{S}_{i}\right)^{\lambda-p_{1}-p_{2}}=0 \quad \text { a.s. }
$$

We now consider the cases $d(B) \geq p\left(\beta_{1}\right)+p\left(\beta_{2}\right)$ and $d(B)<p\left(\beta_{1}\right)+p\left(\beta_{2}\right)$ separately. In the former case, since $\lambda>d(B)$ was arbitrary, (3.13) implies that for any $\eta>0$

$$
P\left(d\left(B \cap B_{\varepsilon, \xi_{1}, \eta}^{+} \cap B_{\varepsilon, \xi_{2}, \eta}^{-}\right) \leq d(B)-p_{1}-p_{2}\right)=1 .
$$

By letting $\eta \downarrow 0$ and noting (3.11), this gives

$$
P\left(d\left(B \cap B_{\beta_{1}}^{+} \cap B_{\beta_{2}}^{-}\right) \leq d(B)-p_{1}-p_{2}\right)=1 .
$$

Since $p_{i}<p\left(\beta_{i}\right)$ are arbitrary, this proves the first part of (3.8). If $d(B)<$ $p\left(\beta_{1}\right)+p\left(\beta_{2}\right)$, then since we chose $\lambda=p_{1}+p_{2},(3.13)$ implies for all $\eta>0$

$$
P\left(B \cap B_{\varepsilon, \xi_{1}, \eta}^{+} \cap B_{\varepsilon, \xi_{2}, \eta}^{-} \neq \varnothing\right)=0 .
$$

Letting $\eta \downarrow 0$ completes the proof of (3.8).

The proof of (3.7) is similar, but easier. Fix $p_{1}<p\left(\beta_{1}\right)$, and if $d(B)<p\left(\beta_{1}\right)$ let $p_{1}$ also satisfy $p_{1}>d(B)$. Choose $\xi_{1}>\beta_{1}$ so that $p_{1}<p\left(\xi_{1}\right)<p\left(\beta_{1}\right)$, and choose $\varepsilon>0$ sufficiently small such that

$$
1-(1+\varepsilon) \xi_{1}^{-1}>1-\beta_{1}^{-1}
$$

and

$$
E\left[Z^{p_{1}}\left(\varepsilon, \xi_{1}\right)\right]<1 .
$$

Next, fix $\lambda>d(B)$ (if $d(B)<p\left(\beta_{1}\right)$ let $\lambda=p_{1}$ ). For $n$ sufficiently large we can choose a cover $\left\{S_{i}\right\}$ of $B$ satisfying (3.12). Define $\left\{\tilde{S}_{i}\right\}$ by

$$
\widetilde{S}_{i}= \begin{cases}S_{i}, & \text { if } S_{i} \cap B_{\varepsilon, \xi_{1}, \eta}^{+} \neq \varnothing, \\ \varnothing, & \text { otherwise. }\end{cases}
$$

By the remarks following Proposition 3.1 we have

$$
P\left(S_{i} \cap B_{\varepsilon, \xi_{1}, \eta}^{+} \neq \varnothing\right) \leq C \rho\left(S_{i}\right)^{p_{1}} .
$$

Thus

$$
E\left[\sum_{i=1}^{\infty} \rho\left(\widetilde{S}_{i}\right)^{\lambda-p_{1}}\right] \rightarrow 0,
$$

and so by Fatou

$$
\liminf _{n \rightarrow \infty} \sum_{i=1}^{\infty} \rho\left(\widetilde{S}_{i}\right)^{\lambda-p_{1}}=0 \quad \text { a.s. }
$$

The remainder of the argument is as before.

Remark. The estimate (3.5) can be used to give a proof (though not necessarily the most elementary one) of (1.6). Fix $t \geq 0$, and note that for any $\beta>1$ and 
$\varepsilon \in(0, \beta-1)$ we can find $p>0$ such that $E\left[Z^{p}(\varepsilon, \beta)\right]<1$. By Proposition 3.1, for any $\eta>0$ there exists a finite constant $C$ such that for all sufficiently small $\zeta$

This implies

$$
P\left([t, t+\zeta] \cap B_{\varepsilon, \beta, \eta}^{+} \neq \varnothing\right) \leq C \zeta^{p} .
$$

and consequently we have

$$
P\left(t \in B_{\varepsilon, \beta, \eta}^{+}\right)=0
$$

$$
P\left(t \in \bigcup_{\eta>0} B_{\varepsilon, \beta, \eta}^{+}\right)=0 .
$$

This means that a.s. for every $\eta>0$ there exists $h \in(0, \eta]$ with $\delta_{t}^{+}(h) \geq$ $h\left(1-(1+\varepsilon) \beta^{-1}\right)$. Thus

$$
P\left(\limsup _{h \downarrow 0} \frac{\delta_{t}^{+}(h)}{h} \geq 1-(1+\varepsilon) \beta^{-1}\right)=1 .
$$

Letting $\beta \uparrow \infty$ completes the proof of (1.6).

4. LOWER Bounds ON $d\left(B \cap B_{\beta}^{+}\right)$AND $d\left(B \cap B_{\beta}\right)$

For $\beta>1, \varepsilon \in(0, \beta-1)$ and $\lambda>0$, define

$$
\tau_{0}(\varepsilon)=\inf \{s \geq 1: W(s)=W(0)\}, \quad X_{0}(\varepsilon, \beta, \lambda)=\tau_{0}(\varepsilon) 1\left(\tau_{0}(\varepsilon) \leq \beta\right),
$$

( $\tau_{0}$ and $X_{0}$ do not actually depend on $\varepsilon$ and $\lambda$, but it simplifies the notation below to include these parameters in their definitions) and for $k \geq 1$

$$
\begin{aligned}
& \tau_{k}(\varepsilon)=\inf \left\{s \geq(1+\varepsilon) \tau_{k-1}(\varepsilon): W(s)=W(0)\right\}, \\
& C_{k}(\varepsilon, \lambda)=\left\{\exists u, v \in\left[\tau_{k-1}(\varepsilon),(1+\varepsilon) \tau_{k-1}(\varepsilon)\right]\right. \\
& \text { with } \left.W(u)=W(0)+\lambda \tau_{k-1}^{1 / 2}(\varepsilon) \text { and } W(v)=W(0)-\lambda \tau_{k-1}^{1 / 2}(\varepsilon)\right\} \text {, } \\
& Y_{k}(\varepsilon, \beta, \lambda)=\frac{\tau_{k}(\varepsilon)}{\tau_{k-1}(\varepsilon)} 1\left(\left\{\frac{\tau_{k}(\varepsilon)}{\tau_{k-1}(\varepsilon)} \leq \beta\right\} \cap C_{k}(\varepsilon, \lambda)\right) \text {, } \\
& X_{k}(\varepsilon, \beta, \lambda)=X_{0}(\varepsilon, \beta, \lambda) \prod_{m=1}^{k} Y_{m}(\varepsilon, \beta, \lambda) \text {, } \\
& X^{*}(\varepsilon, \beta, \lambda)=\sup _{k \geq 0} X_{k}(\varepsilon, \beta, \lambda) .
\end{aligned}
$$

First observe that $\tau_{k}(\varepsilon)<\infty$ a.s. for all $k$. Next, by the scaling and strong Markov properties, $Y_{k}(\varepsilon, \beta, \lambda), k \geq 1$ are i.i.d. with common distribution

$$
\begin{aligned}
& P\left(Y_{1}(\varepsilon, \beta, \lambda) \in[1+\varepsilon, t]\right) \\
& =P(\exists u, v \in[1,1+\varepsilon] \text { with } W(u)=W(1)+\lambda \text { and } W(v)=W(1)-\lambda, \\
& \text { and } \exists s \in[(1+\varepsilon), t] \text { with } W(s)=W(1))
\end{aligned}
$$


for $1+\varepsilon \leq t \leq \beta$, with all remaining mass at the origin. In particular, if $\Rightarrow$ denotes weak convergence, then

$$
Y_{1}(\varepsilon, \beta, \lambda) \Rightarrow Z(\varepsilon, \beta) \text { as } \lambda \downarrow 0 \text {. }
$$

Thus for all $\alpha \geq 0$

$$
E\left[Y_{1}^{\alpha}(\varepsilon, \beta, \lambda)\right] \rightarrow E\left[Z^{\alpha}(\varepsilon, \beta)\right]
$$

Also by the scaling and strong Markov properties $X_{0}(\varepsilon, \beta, \lambda)$ is independent of $\left\{Y_{k}(\varepsilon, \beta, \lambda) ; k \geq 1\right\}$, and hence $X_{k}(\varepsilon, \beta, \lambda)$ is a product of independent random variables.

In what follows we will often consider two independent Brownian motions $W_{1}$ and $W_{2}$ defined on the same probability space $\Omega$. We denote the corresponding random variables in $(4.2)$ by $Y_{k}(i ; \varepsilon, \beta, \lambda), X_{k}(i ; \varepsilon, \beta, \lambda)$, etc. for $i=1,2$. We will use the notation $a_{n} \approx b_{n}$ to mean $a_{n} / b_{n}$ is bounded above and below by finite, nonzero constants which are independent of $n$.

Lemma 4.1. Fix $\delta>0, \beta_{1}>1, \beta_{2}>1$, and assume that $p\left(\beta_{1}\right)+p\left(\beta_{2}\right)<\delta$. Then for all $\varepsilon>0$ sufficiently small there exists $a>0$ and $a p \in(0, \delta)$ such that for all $b \geq \delta$,

$$
E\left[\left(X^{*}\left(1 ; \varepsilon, \beta_{1}, \lambda\right) \wedge X^{*}\left(2 ; \varepsilon, \beta_{2}, \lambda\right) \wedge n\right)^{b}\right] \approx n^{b-p}
$$

where $a \wedge b=\min (a, b)$.

Proof. Let $q_{1}>p\left(\beta_{1}\right)$ and $q_{2}>p\left(\beta_{2}\right)$ satisfy $q_{1}+q_{2}<\delta$. Then by (2.6) and (4.3) for all $\varepsilon>0$ sufficiently small we can find $\lambda>0$ so that $E\left[Y_{1}^{q_{i}}\left(i ; \varepsilon, \beta_{i}, \lambda\right)\right]$ $>1$ for $i=1,2$. So by reducing $q_{1}$ and $q_{2}$ we can find $p_{1}>0$ and $p_{2}>0$ satisfying $p_{1}+p_{2}<\delta$ and

$$
E\left[Y_{1}^{p_{i}}\left(i ; \varepsilon, \beta_{i}, \lambda\right)\right]=1 .
$$

Thus, recalling that $X_{k}\left(i ; \varepsilon, \beta_{i}, \lambda\right)$ is a product of independent random variables, it follows that $X_{k}^{p_{i}}\left(i ; \varepsilon, \beta_{i}, \lambda\right)$ is a nonnegative martingale. Hence $X_{k}^{p_{i}}\left(i ; \varepsilon, \beta_{i}, \lambda\right)$ converges a.s., necessarily to zero, as $k \rightarrow \infty$. Next, for $r>1$ set

$$
T_{r}(i)=\inf \left\{k: X_{k}^{p_{i}}\left(i ; \varepsilon, \beta_{i}, \lambda\right)>r\right\}
$$

Since

$$
X_{T_{r}(i) \wedge k}^{p_{i}}\left(i ; \varepsilon, \beta_{i}, \lambda\right) \leq \beta_{i} r
$$

dominated convergence implies

$$
\begin{aligned}
E\left[X_{0}^{p_{i}}\left(i ; \varepsilon, \beta_{i}, \lambda\right)\right] & =E\left[X_{T_{r}(i) \wedge k}^{p_{i}}\left(i ; \varepsilon, \beta_{i}, \lambda\right)\right] \\
& \rightarrow E\left[X_{T_{r}(i)}^{p_{i}}\left(i ; \varepsilon ; \beta_{i}, \lambda\right) ; T_{r}(i)<\infty\right]
\end{aligned}
$$

as $k \rightarrow \infty$. Observe that on $\left\{T_{r}(i)<\infty\right\}=\left\{X^{*}\left(i ; \varepsilon, \beta_{i}, \lambda\right)^{p_{i}}>r\right\}$ we have

$$
r \leq X_{T_{r}(i)}^{p_{i}}\left(i ; \varepsilon, \beta_{i}, \lambda\right) \leq \beta_{i} r .
$$


Thus

$$
\mu_{i}\left(\beta_{i} r\right)^{-1} \leq P\left(X^{*}\left(i ; \varepsilon, \beta_{i}, \lambda\right)^{p_{i}}>r\right) \leq \mu_{i} r^{-1}
$$

where

$$
\mu_{i}=E\left[X_{0}^{p_{i}}\left(i ; \varepsilon, \beta_{i}, \lambda\right)\right]>0
$$

Now let $p=p_{1}+p_{2}$, and assume that $b \geq \delta$. Then since $p<\delta$

$$
\begin{aligned}
& E\left[\left(X^{*}\left(1 ; \varepsilon, \beta_{1}, \lambda\right) \wedge X^{*}\left(2 ; \varepsilon, \beta_{2}, \lambda\right) \wedge n\right)^{b}\right] \\
& \quad=\int_{0}^{n} b r^{b-1} P\left(X^{*}\left(1 ; \varepsilon, \beta_{1}, \lambda\right)>r\right) P\left(X^{*}\left(2 ; \varepsilon, \beta_{2}, \lambda\right)>r\right) d r \\
& \quad \approx n^{b-p} . \square
\end{aligned}
$$

We will need to consider slightly smaller sets than $B_{\beta}^{+}$and $B_{\beta}^{-}$defined as follows: for $a \geq 1$ let

$$
\begin{aligned}
{ }^{a} \Lambda_{t}^{+}(h) & =\{(s, x): t \leq s \leq t+h, f(t)-a h \leq x \leq f(t)+a h\}, \\
{ }^{a} \delta_{t}^{+}(h) & =\sup \left\{|\Lambda|^{1 / 2}: \Lambda \subseteq{ }^{a} \Lambda_{t}^{+}(h), \Lambda \cap G=\varnothing\right\}, \\
{ }^{a} B_{\beta}^{+} & =\left\{t \in[0,1]: \underset{h \downarrow 0}{\limsup } \frac{{ }^{a} \delta_{t}^{+}(h)}{h} \leq 1-\beta^{-1}\right\},
\end{aligned}
$$

with analogous definitions if + is replaced by - . Observe that since $a \geq 1$, ${ }^{a} \delta_{t}^{+}(h) \geq \delta_{t}^{+}(h)$ and ${ }^{a} \delta_{t}^{-}(h) \geq \delta_{t}^{-}(h)$, hence

$$
{ }^{a} B_{\beta}^{+} \subseteq B_{\beta}^{+} \text {and }{ }^{a} B_{\beta}^{-} \subseteq B_{\beta}^{-} \text {. }
$$

For $i=1,2$, let ${ }^{a} \Lambda_{t}^{+}(i ; h),{ }^{a} \delta_{t}^{+}(i ; h),{ }^{a} B_{\beta}^{+}(i)$ and $B_{\beta}^{+}(i)$ be the quantities analogous to ${ }^{a} \Lambda_{t}^{+}(h),{ }^{a} \delta_{t}^{+}(h),{ }^{a} B_{\beta}^{+}$and $B_{\beta}^{+}$defined relative to $W_{i}$, and similarly with + replaced by - . Define

$$
\mathscr{F}_{i}(t)=\sigma\left\{W_{i}(s): 0 \leq s \leq t\right\}, \quad \mathscr{G}(t)=\sigma\left\{\mathscr{F}_{1}(t) \cup \mathscr{F}_{2}(t)\right\} .
$$

Given a subset $\Gamma$ of $[0,1] \times \Omega$, let $\Gamma(\omega)=\{t \in[0,1]:(t, \omega) \in \Gamma\}$. In what follows we will often write $\Gamma$ for $\Gamma(\omega)$ when the meaning is clear. The following proposition is based on Proposition 14 in [10].

Proposition 4.1. Let $\Gamma$ be a $\mathscr{G}(t)$ progressively measurable subset of $[0,1] \times \Omega$ such that $\Gamma$ is closed a.s. Then for any $a \geq 1$, and $\xi_{1}, \xi_{2}>1$

$$
P\left(\left\{d(\Gamma)>p\left(\xi_{1}\right)+p\left(\xi_{2}\right)\right\} \backslash\left\{\Gamma \cap^{a} B_{\xi_{1}}^{+}(1) \cap^{a} B_{\xi_{2}}^{+}(2) \neq \varnothing\right\}\right)=0 .
$$

Proof. Since the proof of this result is rather lengthy, we divide it into six steps. In the first two steps certain parameters are fixed and various sequences of random variables are defined. Steps 3 and 4 are concerned with some purely geometric arguments used to describe certain events in terms of these random variables. In Step 5 the problem is reduced to equation (4.15), which is then verified in Step 6. 
Step 1. We may assume $p\left(\xi_{1}\right)+p\left(\xi_{2}\right)<1$, else the result is trivial. Fix $\delta \in$ $\left(p\left(\xi_{1}\right)+p\left(\xi_{2}\right), 1\right)$ and let $\beta_{i} \in\left(1, \xi_{i}\right)$ for $i=1,2$ satisfy

$$
p\left(\beta_{1}\right)+p\left(\beta_{2}\right)<\delta \text {. }
$$

Choose $\varepsilon>0, \lambda>0$ and $p \in(0, \delta)$, so that the conclusion of Lemma 4.1 holds, and for $i=1,2$

$$
(1+\varepsilon) \beta_{i} \leq \xi_{i} .
$$

Let $\beta=\beta_{1} \vee \beta_{2}$ and $\beta^{\prime}=\beta_{1} \wedge \beta_{2}$, and choose $M$ so that

$$
M \geq \frac{a \beta^{2}(1+\varepsilon)^{2}}{\beta^{\prime}(1+\varepsilon)-1} \text {. }
$$

Finally, let

$$
\Delta \leq \beta \lambda^{2} M^{-2} .
$$

The parameters $\delta, \beta_{1}, \beta_{2}, p, \varepsilon, \lambda, M$ and $\Delta$ have now been fixed.

Step 2. For $n \geq 1$ (an integer) and $i=1,2$ define

$$
\tau_{0}(i)=\inf \left\{s \geq n^{-1}: W_{i}(s)=W_{i}(0)\right\}, \quad X_{0}(i)=\tau_{0}(i) 1\left(\tau_{0}(i) \leq \beta_{i}\right),
$$

and for $k \geq 1$

$$
\begin{aligned}
\tau_{k}(i)= & \inf \left\{s \geq(1+\varepsilon) \tau_{k-1}(i): W_{i}(s)=W_{i}(0)\right\}, \\
C_{k}(i)= & \left\{\exists u, v \in\left[\tau_{k-1}(i),(1+\varepsilon) \tau_{k-1}(i)\right]\right. \\
& \left.\quad \text { with } W_{i}(u)=W_{i}(0)+\lambda \tau_{k-1}^{1 / 2}(i) \text { and } W_{i}(v)=W_{i}(0)-\lambda \tau_{k-1}^{1 / 2}(i)\right\}, \\
Y_{k}(i)= & \frac{\tau_{k}(i)}{\tau_{k-1}(i)} 1\left(\left\{\frac{\tau_{k}(i)}{\tau_{k-1}(i)} \leq \beta_{i}\right\} \cap C_{k}(i)\right), \\
X_{k}(i)= & X_{0}(i) \prod_{m=1}^{k} Y_{m}(i), \quad X^{*}(i)=\sup _{k \geq 0} X_{k}(i), \quad X^{*}=X^{*}(1) \wedge X^{*}(2) .
\end{aligned}
$$

Then by scaling

$$
n X^{*}(i) \stackrel{d}{=} X^{*}\left(i ; \varepsilon, \beta_{i}, \lambda\right), \quad n X^{*} \stackrel{d}{=} X^{*}\left(1 ; \varepsilon, \beta_{1}, \lambda\right) \wedge X^{*}\left(2 ; \varepsilon, \beta_{2}, \lambda\right) .
$$

Step 3. For any $\eta \leq \Delta$

$$
\begin{aligned}
& \left\{\beta X^{*}>\eta\right\} \subseteq\left\{\forall h \in\left[\beta n^{-1}, \beta^{-2} \eta\right] \exists u(i), v(i) \in\left[h, h(1+\varepsilon) \beta_{i}\right]\right. \\
& \text { with } W_{i}(u(i))=W_{i}(0)+M h \text { and } \\
& \left.W_{i}(v(i))=W_{i}(0)-M h, i=1,2\right\} .
\end{aligned}
$$

To see this, suppose $\beta X^{*}>\eta$, and $h \in\left[\beta n^{-1}, \beta^{-2} \eta\right]$. Then for $i=1,2$ we can find a $k=k(i)$ such that $X_{k}(i)>\beta h$. But this means that for some $m<k$,

$$
h \leq \tau_{m}(i) \leq \beta h
$$


and $C_{m+1}(i)$ occurs. In particular there exist

$$
u(i), v(i) \in\left[\tau_{m}(i), \tau_{m}(i)(1+\varepsilon)\right] \subseteq[h, h(1+\varepsilon) \beta]
$$

such that

$$
\begin{aligned}
& W_{i}(u(i))=W(0)+\lambda \tau_{m}(i)^{1 / 2}, \\
& W_{i}(v(i))=W(0)-\lambda \tau_{m}(i)^{1 / 2} .
\end{aligned}
$$

But by (4.8) and (4.11), since $h \leq \beta^{-2} \eta \leq \beta^{-2} \Delta$

$$
\lambda \tau_{m}(i)^{1 / 2} \geq \lambda h^{1 / 2} \geq M\left(\Delta \beta^{-1}\right)^{1 / 2} h^{1 / 2} \geq M h \beta^{1 / 2} \geq M h
$$

since $\beta>1$. Thus (4.10) holds. The reason for using $\beta X^{*}$ rather than $X^{*}$ on the left-side of (4.10) is that clearly $\left\{X^{*} \leq u\right\} \in \mathscr{G}(\beta u)$, and so $\beta X^{*}$ is a stopping time.

Step 4. Let

$$
\begin{aligned}
& D_{n, \eta}=\left\{t \in \Gamma: \forall h \in\left[\beta n^{-1}, \beta^{-2} \eta\right] \exists u(i), v(i) \in\left[h, h(1+\varepsilon) \beta_{i}\right],\right. \\
& \text { with } W_{i}(t+u(i))=W_{i}(t)+M h \\
& \left.\quad \text { and } W_{i}(t+v(i))=W_{i}(t)-M h, i=1,2\right\},
\end{aligned}
$$

and let $\Theta_{t}$ be the usual shift operator. Then for every $\omega$

$$
\begin{gathered}
\left\{t \in \Gamma: \beta X^{*} \circ \Theta_{t}>\eta\right\} \subseteq D_{n, \eta}, \\
\bigcup_{\eta>0}^{\infty} \bigcap_{n=1}^{\infty} D_{n, \eta} \subseteq \Gamma \cap^{a} B_{\xi_{1}}^{+}(1) \cap{ }^{a} B_{\xi_{2}}^{+}(2) .
\end{gathered}
$$

Assertion (4.12) follows immediately from (4.10). To see (4.13), fix $\eta>0$ and suppose that $t \in \bigcap_{n=1}^{\infty} D_{n, \eta}$. Let $h \in\left(0, \beta^{-2} \eta\right]$, and assume that for either $i=1$ or 2

$$
\Lambda \subseteq^{a} \Lambda_{t}^{+}(i ; h)
$$

where

$$
\Lambda=\left(t, W_{i}(t)\right)+\left(\left[s_{1}, s_{2}\right] \times\left[x_{1}, x_{2}\right]\right)
$$

and

$$
s_{2}-s_{1}=x_{2}-x_{1}=h\left(1-\left((1+\varepsilon) \beta_{i}\right)^{-1}\right) .
$$

We will show that the graph of $W_{i}$ intersects $\Lambda$, and hence

$$
\frac{a_{t}^{+}(i ; h)}{h} \leq 1-\frac{1}{(1+\varepsilon) \beta_{i}} \leq 1-\frac{1}{\xi_{i}},
$$

which implies $t \in \Gamma \cap^{a} B_{\xi_{1}}^{+}(1) \cap{ }^{a} B_{\xi_{2}}^{+}(2)$. To do this, observe that

$$
s_{2} \geq\left(1-\frac{1}{(1+\varepsilon) \beta_{i}}\right) h, \quad s_{1} \leq \frac{1}{(1+\varepsilon) \beta_{i}} h,
$$

and

$$
\frac{1}{(1+\varepsilon) \beta_{i}} s_{2} \geq s_{1} \text {. }
$$


This last fact follows from

$$
\frac{s_{2}}{(1+\varepsilon) \beta_{i}}-s_{1}=\left(1-\frac{1}{(1+\varepsilon) \beta_{i}}\right)\left(\frac{h}{(1+\varepsilon) \beta_{i}}-s_{1}\right) \geq 0 \text {. }
$$

Now let $h^{\prime}=\left((1+\varepsilon) \beta_{i}\right)^{-1} s_{2}$ and note that $s_{1} \leq h^{\prime}<s_{2} \leq h$. Since $t \in$ $\bigcap_{n=1}^{\infty} D_{n, \eta}$ there must exist $u(i), v(i) \in\left[h^{\prime}, h^{\prime}(1+\varepsilon) \beta_{i}\right] \subseteq\left[s_{1}, s_{2}\right]$ with

$$
W_{i}(t+u(i))=W_{i}(t)+M h^{\prime}, \quad W_{i}(t+v(i))=W_{i}(t)-M h^{\prime}
$$

By the definition of $M$ and the previous inequalities we see that $M h^{\prime} \geq a h$, and thus path continuity implies the graph of $W_{i}$ must intersect $\Lambda$. This establishes (4.13).

Step 5. Let

$H_{\eta}=\inf \left\{\sum_{i=1}^{k} \rho\left(S_{i}\right)^{\delta}: \Gamma \subseteq \bigcup_{i=1}^{k} S_{i}, S_{i}\right.$ are closed intervals

$$
\text { with } \left.\rho\left(S_{i}\right) \leq \eta, k \geq 1\right\}
$$

and

$$
E=\left\{H_{\eta} \rightarrow \infty \text { as } \eta \downarrow 0\right\}
$$

Thus

$$
E \supseteq\{d(\Gamma)>\delta\}
$$

Finally let

$$
G_{n, \eta}=\left\{D_{n, \eta} \neq \varnothing\right\} .
$$

In the next step we will show that

$$
P\left(E \backslash \bigcup_{\eta>0} \bigcap_{n} G_{n, \eta}\right)=0 \text {. }
$$

Now clearly $\bigcup_{\eta>0} \bigcap_{n} G_{n, \eta} \supseteq\left\{\bigcup_{\eta>0} \bigcap_{n} D_{n, \eta} \neq \varnothing\right\}$, and since a.s. $D_{n, \eta}$ is compact and $D_{n+1, \eta} \subseteq D_{n, \eta}$

$$
P\left(\bigcup_{\eta>0} \bigcap_{n} G_{n, \eta} \backslash\left\{\bigcup_{\eta>0} \bigcap_{n} D_{n, \eta} \neq \varnothing\right\}\right)=0 \text {. }
$$

Thus by (4.13), (4.14) and (4.15)

$$
P\left(\{d(\Gamma)>\delta\} \backslash\left\{\Gamma \cap^{a} B_{\xi_{1}}^{+}(1) \cap^{a} B_{\xi_{2}}^{+}(2) \neq \varnothing\right\}\right)=0 .
$$

Letting $\delta \downarrow p\left(\xi_{1}\right)+p\left(\xi_{2}\right)$ then completes the proof of the proposition. Thus it remains to prove $(4.15)$.

Step 6. Define

$$
\zeta_{1}=\inf \{t \geq 0: t \in \Gamma\} \wedge 2, \quad \sigma_{1}=\zeta_{1}+\left(\left(\beta X^{*} \circ \Theta_{\zeta_{1}}\right) \wedge 1\right)
$$


and for $k \geq 2$

$$
\zeta_{k}=\inf \left\{t \geq \sigma_{k-1}: t \in \Gamma\right\} \wedge 2, \quad \sigma_{k}=\zeta_{k}+\left(\left(\beta X^{*} \circ \Theta_{\zeta_{k}}\right) \wedge 1\right)
$$

where $\inf (\varnothing)=\infty$. Then $\zeta_{k}$ and $\sigma_{k}$ are stopping times since $\Gamma$ is progressively measurable and $\beta X^{*}$ is a stopping time. Observe also that if $\zeta_{k} \leq 1$, then $\zeta_{k} \in \Gamma$ a.s. since $\Gamma$ is closed a.s. Let

$$
N_{1}=\sup \left\{k: \zeta_{k} \leq 1\right\}
$$

where $\sup (\varnothing)=0$. Then

$$
\Gamma \subseteq \bigcup_{k=1}^{N_{1}}\left[\zeta_{k}, \sigma_{k}\right] \text { a.s. }
$$

Thus by (4.12)

$$
\begin{aligned}
P\left(E \cap G_{n, \eta}^{c}\right) & \leq P\left(E, \max _{1 \leq k \leq N_{1}}\left(\sigma_{k}-\zeta_{k}\right) \leq \eta\right) \\
& \leq P\left(E, \sum_{k=1}^{N_{1}}\left(\sigma_{k}-\zeta_{k}\right)^{\delta} \geq H_{\eta}\right) \\
& \leq P\left(E, H_{\eta} \leq x\right)+P\left(\sum_{k=1}^{N_{1}}\left(\sigma_{k}-\zeta_{k}\right)^{\delta} \geq x\right)
\end{aligned}
$$

for any $x>0$.

We now estimate the last term above. Since $\left(\sigma_{k}-\zeta_{k}\right)={ }^{d} \beta X^{*} \wedge 1$, and $\zeta_{k}$ is independent of $\sigma_{k}-\zeta_{k}$, we have for any $b>0$

$$
\begin{aligned}
E\left[\sum_{k=1}^{N_{1}}\left(\sigma_{k}-\zeta_{k}\right)^{b}\right] & =E\left[\sum_{k=1}^{\infty} 1\left(\zeta_{k} \leq 1\right)\left(\sigma_{k}-\zeta_{k}\right)^{b}\right] \\
& =\sum_{k=1}^{\infty} P\left(\zeta_{k} \leq 1\right) E\left[\left(\sigma_{k}-\zeta_{k}\right)^{b}\right]=E\left[N_{1}\right] E\left[\left(\beta X^{*} \wedge 1\right)^{b}\right] .
\end{aligned}
$$

With $b=\delta$ this implies

$$
E\left[\sum_{k=1}^{N_{1}}\left(\sigma_{k}-\zeta_{k}\right)^{\delta}\right]=E\left[N_{1}\right] E\left[\left(\beta X^{*} \wedge 1\right)^{\delta}\right]
$$

With $b=1$ and the fact that $2 \geq \sum_{k=1}^{N_{1}}\left(\sigma_{k}-\zeta_{k}\right)$ we get

$$
E\left[N_{1}\right] \leq \frac{2}{E\left[\beta X^{*} \wedge 1\right]}
$$

Thus

$$
E\left[\sum_{k=1}^{N_{1}}\left(\sigma_{k}-\zeta_{k}\right)^{\delta}\right] \leq \frac{2 E\left[\left(\beta X^{*} \wedge 1\right)^{\delta}\right]}{E\left[\beta X^{*} \wedge 1\right]}
$$


Now by (4.5), (4.9), and Lemma 4.1, there exists $p \in(0, \delta)$ such that for $b \geq \delta$,

$$
E\left[\left(\beta X^{*} \wedge 1\right)^{b}\right]=n^{-b} E\left[\left(X^{*}\left(1 ; \varepsilon, \beta_{1}, \lambda\right) \wedge X^{*}\left(2 ; \varepsilon, \beta_{2}, \lambda\right) \wedge n\right)^{b}\right] \approx n^{-p} .
$$

This implies that there exists a finite constant $c$ independent of $n$ such that

$$
E\left[\sum_{k=1}^{N_{1}}\left(\sigma_{k}-\zeta_{k}\right)^{\delta}\right] \leq c,
$$

and thus by Chebyshev

$$
P\left(E \cap G_{n, \eta}^{c}\right) \leq P\left(E, H_{\eta} \leq x\right)+c / x .
$$

For any $r>0$ we can choose $x$ sufficiently large and $\eta_{0}$ sufficiently small so that

$$
P\left(E \cap G_{n, \eta_{0}}^{c}\right)<r
$$

for all $n$. Now let $G_{\eta}=\bigcap_{n} G_{n, \eta}$. Since $G_{n+1, \eta} \subseteq G_{n, \eta}$ we have

$$
P\left(E \cap G_{\eta_{0}}^{c}\right)<r .
$$

Equation (4.15) now follows from the fact that $G_{\eta} \uparrow$ as $\eta \downarrow$.

Remark. Let $C \subseteq[0,1]$ be closed. By taking $\Gamma=C \times \Omega$ in Proposition 4.1 we have for every $a \geq 1$

$$
P\left(C \cap^{a} B_{\xi_{1}}^{+}(1) \cap^{a} B_{\xi_{2}}^{+}(2) \neq \varnothing\right)=1
$$

provided $p\left(\xi_{1}\right)+p\left(\xi_{2}\right)<d(C)$. This will be used in the next result.

Theorem 4.1. For every analytic set $B \subseteq[0,1]$ and every $a \geq 1$

$$
\begin{aligned}
& P\left(d\left(B \cap{ }^{a} B_{\beta}^{+}\right) \geq d(B)-p(\beta)\right)=1, \\
& P\left(d\left(B \cap{ }^{a} B_{\beta}^{-}\right) \geq d(B)-p(\beta)\right)=1 .
\end{aligned}
$$

Proof. It suffices to prove the ${ }^{a} B_{\beta}^{+}$case since we obtain the ${ }^{a} B_{\beta}^{-}$case by considering $\widetilde{W}(t)=W(1)-W(1-t)$. We may assume that $p(\beta)<d(B)$, else there is nothing to prove. Since $B$ is analytic, for any $\varepsilon>0$ there exists a compact set $C \subseteq B$ such that $d(C)>d(B)-\varepsilon$. Assume that $\varepsilon$ is sufficiently small that $d(C)>p(\beta)$. Then for any $\beta_{1}$ satisfying $p\left(\beta_{1}\right)<d(C)-p(\beta)$ we have by the remark above

$$
P\left(C \cap^{a} B_{\beta_{1}}^{+}(1) \cap^{a} B_{\beta}^{+}(2) \neq \varnothing\right)=1 .
$$

Thus

$$
P\left(C \cap{ }^{a} B_{\beta_{1}}^{+}(1) \cap{ }^{a} B_{\beta}^{+}(2) \neq \varnothing \mid W_{2}\right)=1 \quad \text { a.s. }
$$

Since for a given $W_{2}$, the random set $C \cap^{a} B_{\beta}^{+}(2)$ may be considered fixed under $P\left(\cdot \mid W_{2}\right)$, and ${ }^{a} B_{\beta}^{+}(1) \subseteq B_{\beta}^{+}(1)$, we can apply (3.7) with $B=C \cap^{a} B_{\beta}^{+}(2)$ to obtain

$$
P\left(d\left(C \cap{ }^{a} B_{\beta}^{+}(2)\right) \geq p\left(\beta_{1}\right) \mid W_{2}\right)=1 \quad \text { a.s. }
$$


Thus

and hence

$$
P\left(d\left(C \cap^{a} B_{\beta}^{+}(2)\right) \geq p\left(\beta_{1}\right)\right)=1
$$

$$
P\left(d\left(B \cap{ }^{a} B_{\beta}^{+}(2)\right) \geq p\left(\beta_{1}\right)\right)=1 .
$$

Since $p$ is continuous, $\beta_{1}$ is arbitrary subject to the requirement that $p\left(\beta_{1}\right)<$ $d(C)-p(\beta)$, and $\varepsilon>0$ is arbitrary, we are done.

Setting $a=1$ in (4.17) together with (3.7) then gives Theorem 2.1. Next we turn to Theorem 2.2.

Theorem 4.2. For every analytic set $B \subseteq[0,1]$ and every $a \geq 1$

$$
P\left(d\left(B \cap{ }^{a} B_{\beta_{1}}^{+} \cap{ }^{a} B_{\beta_{2}}^{-}\right) \geq d(B)-p\left(\beta_{1}\right)-p\left(\beta_{2}\right)\right)=1 .
$$

Proof. We may assume $p\left(\beta_{1}\right)+p\left(\beta_{2}\right)<d(B)$. Fix $\varepsilon>0$ sufficiently small that $p\left(\beta_{1}\right)+p\left(\beta_{2}\right)<d(B)-\varepsilon$. Choose $C \subseteq B$ compact such that $d(C)>d(B)-\varepsilon$, and let $\beta_{3}$ satisfy

$$
p\left(\beta_{1}\right)+p\left(\beta_{2}\right)+p\left(\beta_{3}\right)<d(C) .
$$

Arguing as in Theorem 4.1, it suffices to show

$$
P\left(C \cap{ }^{a} B_{\beta_{1}}^{+}(1) \cap{ }^{a} B_{\beta_{2}}^{-}(1) \cap{ }^{a} B_{\beta_{3}}^{+}(2) \neq \varnothing \mid W_{1}\right)=1 \text { a.s. }
$$

(let $B=C \cap^{a} B_{\beta_{1}}^{+}(1) \cap{ }^{a} B_{\beta_{2}}^{-}(1)$ in (3.7), etc.). To prove this we will show that if $\eta$ is sufficiently small, then

$$
P\left(C \cap^{a} B_{\beta_{1}}^{+}(1) \cap^{a} B_{\beta_{2}, \eta}^{-}(1) \cap^{a} B_{\beta_{3}}^{+}(2) \neq \varnothing\right)=1
$$

where

$$
{ }^{a} B_{\beta_{2}, \eta}^{-}(1)=\left\{t \in[0,1]: \frac{{ }^{a} \delta_{t}^{-}(1 ; h)}{h} \leq\left(1-\beta_{2}^{-1}\right) \text { for all } h \in(0, \eta]\right\} .
$$

Since ${ }^{a} B_{\beta_{2}, \eta}^{-}(1) \subseteq{ }^{a} B_{\beta_{2}}^{-}(1)$, (4.20) then follows.

In order to prove (4.21) all we need to do is show that

$$
P\left(d\left(C \cap \cap^{a} B_{\beta_{2}, \eta}^{-}(1)\right)>p\left(\beta_{1}\right)+p\left(\beta_{3}\right)\right)=1
$$

and apply Proposition 4.1 ( $\Gamma$ defined by $\Gamma(\omega)=C \cap{ }^{a} B_{\beta_{2}, \eta}^{-}(1)$ is a.s. closed and progressively measurable). To do this choose $\beta<\beta_{2}$ such that $p\left(\beta_{1}\right)+$ $p(\beta)+p\left(\beta_{3}\right)<d(C)$. By Theorem 4.1, since $d(C)-p(\beta)>p\left(\beta_{1}\right)+p\left(\beta_{3}\right)$,

$$
P\left(d\left(C \cap{ }^{a} B_{\beta}^{-}(1)\right)>p\left(\beta_{1}\right)+p\left(\beta_{3}\right)\right)=1 .
$$

But $\lim _{\eta \downarrow 0}{ }^{a} B_{\beta_{2}, \eta}^{-}(1) \supseteq{ }^{a} B_{\beta}^{-}(1)$, and hence

$$
\lim _{\eta \downarrow 0} d\left(C \cap^{a} B_{\beta_{2}, \eta}^{-}(1)\right) \geq d\left(C \cap^{a} B_{\beta}^{-}(1)\right)>p\left(\beta_{1}\right)+p\left(\beta_{3}\right) \quad \text { a.s. }
$$

Thus (4.22) holds.

Combining the results in Theorem 3.1 and Theorem 4.2 with (4.4) we see that for every analytic set $B \subseteq[0,1]$ and every $a \geq 1, B \cap{ }^{a} B_{\beta}^{+} \cap{ }^{a} B_{\beta}^{-}=\varnothing$ a.s. if $2 p(\beta)>d(B)$, while if $2 p(\beta) \leq d(B)$ then

$$
P\left(d\left(B \cap{ }^{a} B_{\beta}^{+} \cap{ }^{a} B_{\beta}^{-}\right)=d(B)-2 p(\beta)\right)=1 .
$$


Thus to complete the proof of Theorem 2.2 it suffices to prove

Proposition 4.2. If $a \geq 2 \beta(\beta-1)^{-1}$ then for every $\omega$

$$
{ }^{a} B_{\beta}^{+} \cap{ }^{a} B_{\beta}^{-} \subseteq B_{\beta} \subseteq B_{\beta}^{+} \cap B_{\beta}^{-} .
$$

Proof. Clearly $B_{\beta} \subseteq B_{\beta}^{+} \cap B_{\beta}^{-}$. To prove the other inclusion we argue by contradiction. Thus assume that $t \in\left({ }^{a} B_{\beta}^{+} \cap{ }^{a} B_{\beta}^{-}\right) \backslash B_{\beta}$. Since $a \geq 1, t \in$ $\left(B_{\beta}^{+} \cap B_{\beta}^{-}\right) \backslash B_{\beta}$. Hence there exists a sequence $h_{n} \downarrow 0$ and a sequence of squares $\Lambda_{n}$ such that

$$
\begin{gathered}
\Lambda_{n} \subseteq \Lambda_{t}\left(h_{n}\right) \backslash G, \\
\Lambda_{n} \cap \Lambda_{t}^{+}\left(h_{n}\right) \neq \varnothing, \quad \Lambda_{n} \cap \Lambda_{t}^{-}\left(h_{n}\right) \neq \varnothing, \\
\left|\Lambda_{n}\right|^{1 / 2} h_{n}^{-1} \geq \nu>1-\beta^{-1} .
\end{gathered}
$$

By taking a further subsequence if necessary we may assume that at least half of $\Lambda_{n}$ intersects $\Lambda_{t}^{+}\left(h_{n}\right)$, i.e.

$$
\left|\Lambda_{n} \cap \Lambda_{t}^{+}\left(h_{n}\right)\right|^{1 / 2} h_{n}^{-1} \geq \nu / \sqrt{2},
$$

(if not, argue using $\Lambda_{t}^{-}\left(h_{n}\right)$ in place of $\Lambda_{t}^{+}\left(h_{n}\right)$ in what follows). But then by the definition of $a$,

$$
{ }^{a} \delta_{t}^{+}\left(\frac{\nu h_{n}}{2}\right) \geq \frac{\nu h_{n}}{2}
$$

which contradicts $t \in{ }^{a} B_{\beta}^{+}$.

Acknowledgment. We would like to thank Dan Waterman for bringing this problem to our attention.

\section{REFERENCES}

1. M. T. Barlow and E. A. Perkins, Brownian motion at a slow point, Trans. Amer. Math. Soc. 296 (1986), 741-775.

2. B. Davis, On Brownian slow points, Z. Wahrsch. Verw. Gebeite 64 (1983), 359-367.

3. B. Davis and E. A. Perkins, Brownian slow points: the critical case, Ann. Probab. 13 (1985), 779-803.

4. A. Denjoy, Lecons sur le calcul des coefficients d'une serie trigonometrique, Part II, Metrique et topologie d'ensembles parfait et de fonctions, Gauthier-Villars, Paris, 1941.

5. E. P. Dolzhenko, Boundary properties of arbitrary functions, Math. USSR 12 (1967), no. 1, $1-12$.

6. J. Foran, Continuous functions need not have $\sigma$-porous graphs, Real Anal. Exchange 11 (1985-86), 194-203.

7. C. Goffman, The graph of Brownian motion is not strongly porous, Preprint, 1988.

8. P. A. Greenwood and E. A. Perkins, A conditioned limit theorem for random walk and Brownian local time on square root boundaries, Ann. Probab. 11 (1983), 227-261.

9. __ Limit theorems for excursions from a moving boundary, Theor. Probab. Appl. 29 (1984), 517-528. 
10. E. Perkins, On the Hausdorff dimension of the Brownian slow points, Z. Wahrsch. Verw. Gebiete 64 (1983), 369-399.

11. B. S. Thomson, Real functions, Springer-Verlag, New York, 1985.

12. L. Zajicek, Sets of $\sigma$-porosity and sets of $\sigma$-porosity (q), Časopis Pěst. Mat. 101 (1976), 350-359.

Department of Mathematics, Syracuse University, Syracuse, New York 13244-1150

E-mail address (J. T. Cox): jtcox@rodan.acs.syre.edu 